\title{
ESTUDO DE PLACAS CIMENTÍCIAS PARA USO EM HABITAÇÕES POPULARES: UMA REVISÃO SISTEMÁTICA DA LITERATURA
}

\section{CEMENT BONDED FOR USE IN POPULAR HOUSING: A SYSTEMATIC REVIEW OF THE LITERATURE}

\section{T. L. PILZ ${ }^{1, *}$, J. R. S. FERREIRA ${ }^{2}$, J. S. M. NEGRÃO ${ }^{1}$, N. C. SOTSEK ${ }^{1}$ and T. GENARO ${ }^{1}$}

${ }^{1}$ Federal University of Paraná, Department of Production Engineering, Curitiba, Paraná, Brazil

${ }^{2}$ Federal University of Paraná, Department of Civil Engineering, Curitiba, Paraná, Brazil

${ }^{*}$ Corresponding author. Federal University of Paraná, Department of Production Engineering, Curitiba, Paraná, Brazil, Phone: +5547 991566085

e-mail address: thaisa.pilz@gmail.com (T.L. Pilz).

\section{A R T I C L E I N F O}

Article history:

Received 2019-12-03

Accepted 2020-01-03

Available online 2020-12-20

\section{palavras-chave}

Placas cimentícias

5W1H

Revisão Sistemática da Literatura

Habitação Popular

keywords

Cement Bonded

5W1H

Literature Review

Popular Housing
A B S T R A C T

This work deals with the element of popular house sealing using recyclable materials. The objective of this research was to develop a systematic review in order to identify impact research to understand the technique and application of panels that use recyclable materials in dwellings. This objective includes the identification of the materials used, the traces of the mixtures, the results of tests (resistance, water absorption, etc.), time for the research and whether or not there is possibility or future use in popular housing. The focus of the study is to look for articles that make possible the artisanal production, once the goal is that in the future the villager and the volunteers can produce as much of the house as possible with their own hands. A Systematic Review of Literature was carried out in the electronic databases, searching bibliographies in both English and Portuguese, covering the articles, magazine and congress. The selection and analysis of the scientific works and the extraction of data according to the $5 \mathrm{~W} 1 \mathrm{H}$ tool were made. This is a summary of the general idea of the articles selected that have great relevance in the research. The results appear to be valid, promising traits have been achieved, however, further studies and development of the plaques are required.

R E S U M O

Este trabalho trata do elemento de vedação de moradias populares aproveitando materiais recicláveis. $O$ objetivo desta pesquisa foi desenvolver uma revisão sistemática a fim de identificar pesquisas de impacto para o entendimento da técnica e de aplicação dos painéis que utilizam materiais recicláveis em habitações. Dentro deste escopo se incluem a identificação dos materiais utilizados, os traços das misturas, os resultados de testes (resistência, absorção de água, etc.), tempo destinado a execução da pesquisa e se há ou não possibilidade ou utilizá-las futuramente em projetos de habitações populares. O foco do estudo é buscar artigos que possibilitem a produção artesanal, isto é, o propósito é que no futuro o próprio morador e os voluntários possam produzir o máximo possível da moradia com as próprias mãos. Foi realizada uma Revisão Sistemática da Literatura nas bases de dados eletrônicas, buscando bibliografias tanto em inglês como em português, cobrindo os artigos, de revista e de congresso. Foi feita a seleção e análise dos trabalhos científicos e a extração de dados segundo a ferramenta 5 WIH. Trata-se de um resumo da ideia geral dos artigos selecionados que apresentam grande relevância na pesquisa. Os resultados parecem válidos, chegou-se a traços promissores, entretanto são necessários novos estudos e prosseguimento com o desenvolvimento das placas. 


\section{INTRODUÇ̃̃̃O}

Com o aumento da população, cresce também a demanda de recursos. Se a relação entre a taxa de utilização e a capacidade de sustentação não for equilibrada resulta em crescimento da geração de resíduos e consequente aumento da poluição. Com isso, toda a forma de reciclagem é bem-vinda. Na construção civil, ramo que é responsável por grande parte do consumo de recursos naturais, muito se estuda sobre formas de reutilizar estes resíduos como substitutos ou compósitos para materiais de construção sustentáveis. A valorização desses materiais alternativos como opção ao convencional deve proporcionar um produto de qualidade, estética, e potencialmente redutor de impactos ambientais (Braga et al, 2005).

As placas cimentícias, peças pré-fabricadas que atuam basicamente na vedação de construções, apresentam vantagens sobre os outros materiais de mesma função, como os blocos. A praticidade, rapidez e limpeza são as principais vantagens dessa construção a seco, que exige muito menos habilidade de quem vai montá-las comparado ao preparo necessário para um pedreiro de uma obra "convencional". Com isso, percebe-se que essa é uma ótima alternativa para construção de moradias para a população de baixa renda, uma vez que, na maioria das vezes quem as constrói são voluntários e/ou os próprios moradores, os quais geralmente apresentam pouca ou nenhuma prática na construção civil (Nogueira, 2014).

O caso da América Latina e Caribe ilustra que a diferença proporção da população urbana frente à rural passou de $68 \%$ em 1985 para $76 \%$ em 2005. Este movimento, somado ao crescimento populacional, e o modo de consumo deste período levou a um aumento de $60 \%$ na população urbana da região em 20 anos (AIDIS, 2006). No período de 1980 a 2005, a quantidade de RSU per capita aumentou $29 \%$ na América do Norte, 35\% na OCDE, e 54\% na UE15, acompanhando, de uma forma geral, o produto interno bruto (PIB) dos países (Sjöstöm e Östblom, 2010). Visando a solução deste problema, o presente artigo tem como foco uma revisão sistemática da literatura à respeito da possibilidade de usar resíduos em geral na produção de placas de cimento que possam ser utilizadas na construção civil, verificando suas propriedades e sempre com ligação à projetos sustentáveis e/ou de baixo custo.

Tabela 1 - Palavras-Chave (português e inglês). Palavras-chave

Placas cimentícias

Reciclagem

Moradia

Sustentabilidade

Baixo custo

Resíduos

Popular

Social

Compósito

Traço

\section{REVISÃO DE LITERATURA}

O crescimento e desenvolvimento da população mundial e o aumento de suas necessidades elevam consigo os níveis de poluição e a geração de resíduos. A única forma de desenvolvimento que faz algum sentido é o desenvolvimento sustentável (Solheim, 2018). Inúmeros estudos vêm sendo feitos a fim de diminuir os traumas ambientais. Os resíduos da construção civil e demolição correspondem a 50\% de todos os resíduos sólidos urbanos e é comumente despejado em lugares impróprios (Contreras et al., 2016). Tendo isso em vista, muito se fala em projetos sustentáveis e diversos estudos são realizados para que haja a possibilidade de agregar os mais variados tipos de materiais reciclados em compósitos e demais utensílios da construção.

Simultaneamente, por serem alternativas de baixo custo, pensou-se em utilizar esses materiais no desenvolvimento de habitações populares, oferecendo assim melhor qualidade de vida à pessoas de baixa renda. Visintainer et al. (2012) buscaram, em projeto, por materiais sustentáveis e baratos que, juntamente com uma forma de construção inteligente que una elementos naturais e sustentáveis, atuem na concepção de casas para um padrão de vida pouco elevado, já que há a possibilidade de construir moradias populares (com poucos recursos financeiros) levando em conta questões ambientais.

O modelo de "dry construction", ou construção à seco, é um sistema prático, barato e limpo. Alguns estudos buscam a produção de placas cimentícias utilizando materiais alternativos sustentáveis, como o de Fuwape et al. (2007), que utilizou resíduos de papel e serragem e prova que essa, assim como outras placas feitas com os mais diversos materiais apresentam resistência, boa aparência e são boas opções para a construção civil, com ótimo custo-benefício além do resguardo ambiental.

\section{MÉTODO DE PESQUISA}

O método utilizado para desenvolver o presente artigo foi a revisão sistemática de literatura que pode ser utilizada com a finalidade de aumentar o desempenho de pesquisas ou avaliar suas tendências, investigando as características das publicações, como por exemplo: autoria, fontes de pesquisa, temas e citações (Small, 2003).

\section{Keywords}

Cement plate, cement slab, cement panel, cement bonded*, cement board, cement sheet, cement particleboard. (* apresentou o maior número de resultados.)

Recycle

Housing

Sustainability

Low cost

Waste

Popular

Social

Composite

Ratio 
Esses termos, utilizados separadamente e combinados, como por exemplo: Placas cimentícias e sustentabilidade, moradia e baixo custo, entre outros, possibilitaram a obtenção de 87 artigos. O primeiro passo da análise, após as pesquisas, foi excluir resultados exibidos pelas bases, por meio de uma análise superficial delas, uma vez que não estavam de acordo com os critérios previamente determinados, ou seja, artigos que não se relacionam ao tema foram descartados. Além disso, foi possível, nessa fase, eliminar alguns artigos repetidos.

Por meio da análise do resumo e conclusão do texto, foram descartados artigos que não se enquadraram aos requisitos da revisão, por exemplo, resultados com materiais não destinados à moradia (como os testados para vedar tanques de água), artigos sobre reforço das placas com fibras e não sobre as placas em si, entre outros que fogem ao tema e reduzem o número de material encontrado que se adequa. Com a leitura completa do estudo, criou-se uma tabela com as informações principais (Tabela 3) dos artigos selecionados filtrando os artigos realmente relevantes à pesquisa e excluindo o restante dos estudos.

Após a filtragem, foram identificados 9 artigos em que seriam relevantes ao desenvolvimento da pesquisa. Para a extração dos dados destes documentos foi utilizada a ferramenta $5 \mathrm{~W} 2 \mathrm{H}$, porém o termo "HOW MUCH" foi desconsiderado na análise por não ter uma abordagem clara quanto aos custos. Essa ferramenta permite a rápida identificação de elementos principais e essenciais de um projeto. Para cada um dos estudos foram feitas as perguntas da Tabela 2.
Tabela 2 - Significado da tabela 5W1H nessa pesquisa.

5W1H Perguntas

WHO

Quem desenvolveu o artigo.

WHAT

Ensaios realizados e traço utilizado.

WHERE

WHY

Local de aplicação da placa.

WHEN

Por que esse tipo de material foi escolhido.

HOW

Quando foi publicado o artigo.

Material que foi utilizado.

A análise utilizando a ferramenta $5 \mathrm{~W} 1 \mathrm{H}$ foi feita seguindo a ordem da Tabela 2, propiciando, assim, uma linha de informações a fim de facilitar o entendimento imediato do texto. Escolheu-se esse método a fim de obter uma visão panorâmica dos seguintes elementos utilizados em placas cimentícias: os materiais utilizados, os traços das misturas, os resultados de testes (resistência, absorção de água, etc.) e o tempo destinado, a fim de verificar se há a possibilidade de utilizar estes parâmetros em um futuro projeto de habitações populares no Brasil.

O item "WHAT", nessa revisão, foi considerado em dois tópicos: os ensaios que foram realizados e o traço utilizado na fabricação das placas. "WHERE" é composto pelos locais onde as placas foram aplicadas, por exemplo: em certos estudos as placas foram utilizadas em fachadas ou divisórias, em outros como forros e etc. O plano inicial era inserir, no item "WHEN", o tempo de duração da pesquisa, testes e conclusão do trabalho. No entanto essa informação não estava presente na maior parte dos artigos e então optou-se pela data de publicação deles.

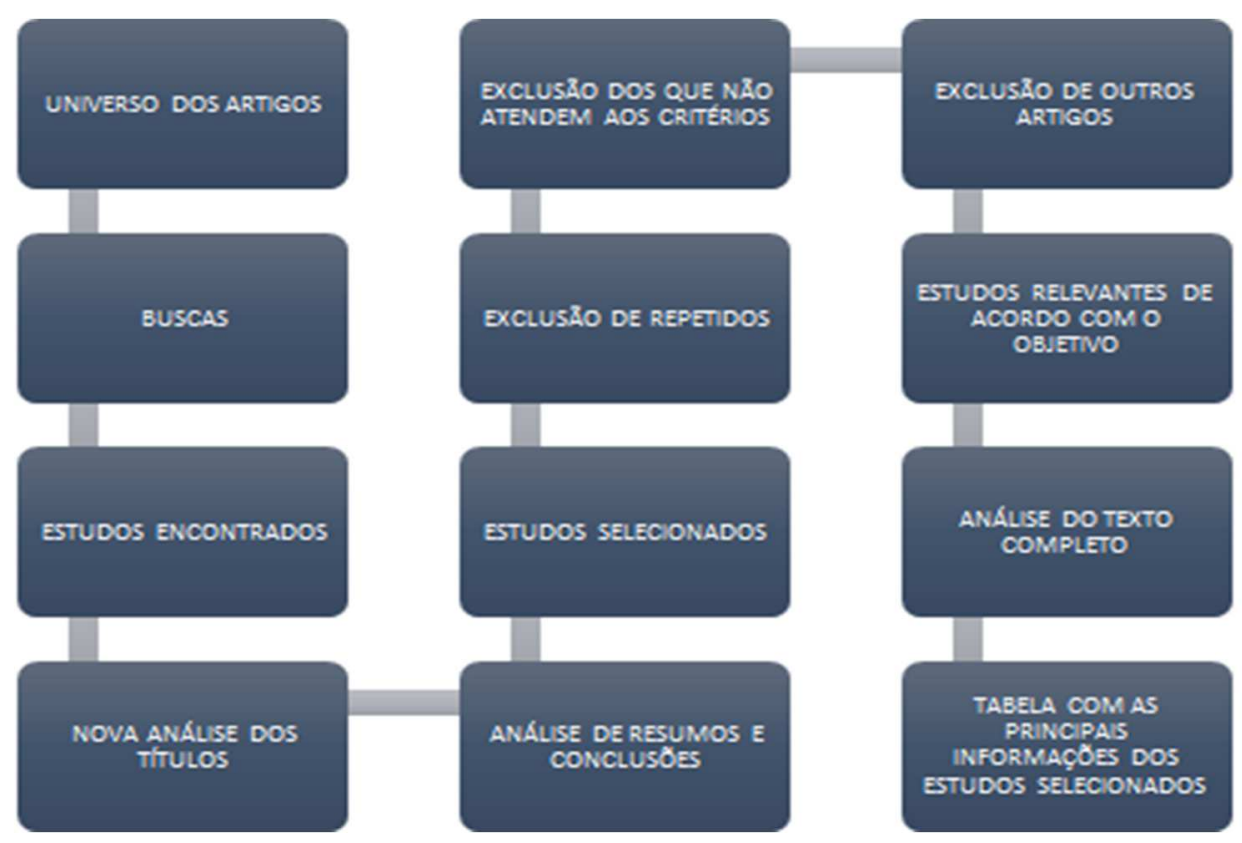

Figura 1 - Esquema das Etapas da Revisão Sistemática.

\section{RESULTADOS E DISCUSSÃO}

Neste item são apresentados, resumidamente, os estudos que foram selecionados e quais foram seus autores, objetivos e conclusões, bem como a Tabela 3 com a síntese obtida pelo uso da ferramenta adaptada, $5 \mathrm{~W} 1 \mathrm{H}$.

4.1 Placas cimentícias à base de resíduos sólidos domiciliares

AUTORES: João Marcos Bosi Mendonça de Moura e Lúcio Flávio da Silveira Matos 
OBJETIVO: avaliar parâmetros físicos e mecânicos de placas cimentícias alternativas, à base de resíduos sólidos domiciliares, compostos por polímeros, papéis e vidro. Realizaram-se ensaios de determinação da resistência à compressão, resistência à flexão, de massa específica aparente, absorção de água e de microscopia eletrônica de varredura. Os resultados foram comparados com os dados técnicos das placas cimentícias convencionais (sem amianto) e das placas de EPS, disponíveis na literatura. Concluiu-se que todos os índices obtidos para o compósito formado à base de resíduos proveniente da coleta seletiva do município de Blumenau-SC alcançaram os limites mínimos necessários dos parâmetros abordados, para sua aplicação em estruturas de vedação.

CONCLUSÃO: Os resultados permitiram de corroborar quatro hipóteses: (1) que a incorporação de RSD (resíduos sólidos domiciliares) proporcionou um significativo aumento na elasticidade da placa, tendo em vista o modo como ela se rompeu nos ensaios de resistência à compressão e flexão. Isto se deve, possivelmente, pela suficiente aderência entre o cimento, a massa acrílica e os resíduos sólidos domiciliares de formato particulado; (2) a massa específica aparente foi mais baixa que a das placas cimentícias convencionais, possivelmente devido a presença dos resíduos. (3) Destaca-se que os padrões normativos e técnicos referentes à resistência à compressão e à absorção de água foram atendidos. (4) Através da análise de microscopia eletrônica de varredura, os polímeros PET e EPS parecem adquirir uma melhor ligação com a pasta cimentícia, se comparado ao que acontece com a zona de contato entre a pasta e resíduos fibrosos. Neste último caso, observou-se a formação de vazios, o que pode influenciar parâmetros fundamentais, aumentando a absorção de água e diminuindo a resistência mecânica.

4.2 Revestimento cimentício composto por resíduos da construção civil e lama de mármore e granito

AUTORES: Ana Claudia Marques, Alessandro Campos, Rosemary Matias, Suellen Regina de Oliveira Barreto.

OBJETIVOS: Este trabalho tem como objetivo produzir um revestimento cimentício de parede, que tenha, na sua composição, resíduos da construção civil (RCC), tipo A (lama de mármore e granito), e resíduos provenientes de beneficiamento de rochas ornamentais (mármores e granitos). Este trabalho é derivado de uma pesquisa de graduação onde se discutiu o destino dos resíduos da construção civil no município de Campo Grande, MS. Levando em consideração que o Brasil tem a construção civil como o segundo maior PIB, podemos também entender que a alta produção e serviço dentro deste campo é incontestável, portanto, implementar formas de reutilização destes materiais é contribuir para a economia e também para o meio ambiente. Foram realizadas coletas em cooperativas e marmorarias locais, onde foi obtida a obra prima já em forma de agregado e depois todos os processos de montagem e forma das peças foram gerados no laboratório de materiais da Universidade Católica Dom Bosco, assim como os testes de rompimento das mesmas. As peças tiveram um resultado interessante, no que diz respeito a acabamento e porosidade, mas ainda precisa passar por novos processos para ter uma superfície mais lisa esteticamente aceitável pelo mercado, uma vez que se deseja que ela seja usada para revestimento de paredes.

CONCLUSÃO: As placas cimentícias apresentaram resultados satisfatórios em sua estabilização, seu acabamento superficial ainda se apresenta poroso, por conta do empacotamento dos grãos de RCC e RMG, e devido ao método de produção, com compactação manual da mistura. No entanto novas etapas na pesquisa deste produto como testes de resistência à compressão e testes de absorção de água, assim como testes de desempenhos térmicos e desempenhos acústicos, precisam ser realizadas para verificar melhor acabamento e desempenho técnico segundo as normas vigentes e melhor aceite pelo mercado.

\subsection{Resistência mecânica de compósitos cimentícios leves utilizando resíduos industriais e fibras de sisal}

AUTORES: Nivaldo T. de Arruda Filho, Carolina P. Dantas, Antonio F. Leal, Normando P. Barbosa, Cibelle G. Silva; Mavinieux V. Alexandre.

OBJETIVO: desenvolver elementos construtivos para forros e divisórias, a partir de matrizes cimentícias com incorporação de resíduos industriais (resíduo cerâmico, etil vinil acetato - EVA) e fibras de sisal. Desenvolveram-se técnicas de moldagem em matrizes cimentícias auto adensáveis e se avaliou a resistência mecânica dos novos compósitos. Uma placa com resíduos de EVA foi produzida e, através de trabalhos de reologia, encontrou-se a pasta matriz de revestimento desta placa, com teor adequado de adições pozolânicas e aditivo superplastificante. Avaliaram-se as resistências mecânicas das placas, da pasta matriz de revestimento encontrada, com e sem adição de fibras, e do novo compósito formado pela união desses dois elementos. Utilizou-se a técnica de alinhamento de fibras com o intuito de incrementar resistência ao novo compósito leve. A adição da matriz com fibras alinhadas melhorou a resistência a flexão do novo compósito.

CONCLUSÃO: Os resultados dos ensaios de resistência a compressão simples indicaram que substituição pozolânica (metaculinita e tijolo moído) aumentaram o comportamento mecânico. 2. O melhor comportamento da pasta matriz de revestimento é observado após 28 dias de cura em ambiente úmido. 3. A incorporação das fibras a pasta de revestimento da placa de etil vinil acetato (EVA), sejam elas distribuídas de forma randômica ou alinhadas dentro da pasta, acrescentaram resistência à flexão ao novo compósito quando comparada com a placa de EVA revestida com a pasta de matriz sem adição de fibras. 4. As fibras distribuídas de forma alinhadas dentro da pasta de revestimento, incrementaram resistência á flexão ao compósito (pasta matriz + placa de EVA) sendo esta, superior ao compósito com a pasta matriz de revestimento com fibras randômicas e sem fibras.

4.4 Plates made with solid waste from the recycled paper industry (placas feitas com resíduos sólidos de indústrias de papel reciclável) Valle.

AUTORES: M. Pelegrini, I. Gohr Pinheiro, J.A.B.

OBJETIVOS: Os resultados de ensaios realizados em chapas utilizadas na indústria da construção, inteiramente fabricados com resíduos sólidos de uma indústria de papel reciclado, são apresentados e comparados com os resultados obtidos com chapas de madeira. Resultados anteriores mostraram que os resíduos são compostos essencialmente de polímeros quando estes resíduos são gerados por papel reciclado produzido com as aparas "tipo II”. Esses resíduos sólidos foram colocados em um molde que foi aquecido e prensado com uma prensa hidráulica para obter as placas. As placas produzidas 
foram submetidos aos testes de umidade, inchamento, absorção de água, densidade, módulo de flexão estática à ruptura, módulo de elasticidade e retirada direta do parafuso. Esses mesmos ensaios foram realizados em dois tipos de placas comerciais de madeira, madeira aglomerada e compensada, a fim de compara os resultados com os obtidos com a placa de resíduos. As placas de resíduos apresentaram comportamento semelhante ao da placa de madeira aglomerada, mas foi possível distinguir maior flexibilidade na placa de resíduos e uma diferença significativa nos testes de inchamento e absorção de água que mostraram que a placa de resíduos teve melhor desempenho que a madeira aglomerada e placas de contraplacado.

CONCLUSÃO: Os resíduos estudados nessa pesquisa são gerados em grandes quantidades no Brasil. O desperdício em questão é descartado tornando- se um grande problema ambiental quando poderia ser usado no lugar de matéria prima para diversas aplicações. Conhecendo a composição dos resíduos (composto basicamente por polímeros), foi possível determinar os valores de resistência e densidade das placas, chegando a resultados semelhantes aos das placas de madeira testadas, trazendo algumas vantagens sobre elas como: baixa absorção de água e excelente flexibilidade. A proposta foi realizada com um controle rigoroso nos materiais que compuseram a placa. Outro ponto positivo é a possibilidade de fabricá-las em escala industrial uma vez que o equipamento que se faz preciso já é uma tecnologia conhecida. Caso a produção das placas seja feita próxima ao local do aterro muito dinheiro seria poupado. O aspecto sustentável ainda atrairia atenções mundiais.

Tabela 3 - Síntese dos dados pela utilização da ferramenta 5W1H.

\section{WHO}

\section{WHAT} WHERE

Vedação

4.1 Moura

Resistência à compressão, à

flexão, massa específica aparente e absorção de água

1:1:1:0,2 massa acrílica: papéis: polímeros tipo 1 (PET e PP): polímeros tipo 2 (EPS)

4.2 Marques et al.

4.3 Arruda Filho et al.

Estabilização da mistura, acabamento superficial; desmolde, verificar se nãose decompõe; Compressão simples

1:3 (1 parte de cimento:3 partes de RCC), 1:1,5:1,5 (1 parte de cimento: 1,5 partes de RCC: 1,5 partes de RMG), $1: 2,25: 0,75$

Resistência à compressão, resistência à flexão

As placas de EVA foram produzidas no traço 1:6 (cimento: agregado EVA)

4.4 Pelegrini, Testes de umidade, Pinheiro inchamento, absorção de água, e Valle densidade, resistência à flexão, módulo de elasticidade $1 \mathrm{~kg}$ de resíduo. pressão de $10,2 \mathrm{kPa}$ e temperatura de $150^{\circ} \mathrm{C}$ por $2 \mathrm{~h}$.

4.5 Fuwape, Módulo de ruptura, módulo de Fabiyi e elasticidade, absorsão de água, Osuntuyi inchamento de espessura relação cimento / partícula de 3,0: 1 e 3,5: 1 são adequados para construção civil
Revestimento Alternativa sustentável cimentício para aplicação interna e externa de paredes para a reutilizaçao de resíduos da construção civil (RCC), tipo A, e resíduos provenientes de beneficiamento de rochas ornamentais (mármores e granitos).

Forro e divisórias

reduzir impactos da poluição ambiental.

Não fala, compara com placas de madeira

Divisórias, teto e paineis
A reciclagem de papel impede seu acúmulo em aterros sanitários, gasta menos energia, gera renda e diminui o consumo de recursos naturais

Reaproveitamento de resíduos de papel e serragem que seria queimada ou jogada em aterros.
WHEN

HOW

2015

Polímeros (PET, PP e EPS), papéis, vidro
2017

RCC do tipo A e a lama de mármore e de granitos (RMG)
2012 Resíduos industriais (resíduo cerâmico, etil vinil acetato EVA) e fibras de sisal

2009 Polímeros, componentes dos resíduos sólidos da reciclagem de papeis

Resíduos de papel e serragem 
4.5 Technical assessment of three layered cement-bonded boards produced from wastepaper and sawdust (avaliação técnica de três placas de cimento produzidas com resíduos de papel e serragem)

AUTORES: Joseph Adeola Fuwape, James Sunday Fabiyi, Edward Olusola Osuntuyi.

OBJETIVOS: As propriedades técnicas de três placas de cimento feitas com resíduos de papel e serragem foram investigadas. As placas foram produzidas em três níveis de densidade: 1000,1200 e $1300 \mathrm{~kg} / \mathrm{m}^{3}$. e em quatro traços de relação cimento/ partícula: 2,0: 1, 2,5: 1, 3,0: 1 e 3,5: 1. As propriedades técnicas avaliadas foram: módulo de elasticidade, módulo de ruptura, absorção de água e inchamento de espessura. Chegou se à conclusão que as relações cimento/ partícula 3,0: 1 e 3,5: são adequadas para construção civil, como painéis, tetos e coberturas divisórias mesmo em lugares de aplicação com temperaturas extremas.

CONCLUSÃO: a produção de placas de cimento de papel usado e serragem satisfez a especificação placas de densidade moderada com base nos dados obtidos neste estudo.

A Tabela 3 organiza os artigos mais relevantes destes temas, selecionados pelos critérios descritos no item 3 deste trabalho, além de resumir as principais informações de cada artigo de uma forma visualmente agradável, facilitando o entendimento.

\section{CONCLUSÃO}

A geração excessiva de resíduos tem graves consequências ambientais e sociais. A reutilização desses materiais é extremamente importante e pertinente no cenário de crescimento populacional. Atualmente, muitos estudos são feitos em prol da sustentabilidade, visando modificar materiais usados na construção civil. As mudanças buscam manter sua qualidade, mas ao mesmo tempo reduzir os impactos ambientais causados. Junto a isso, buscam-se alternativas de baixo custo para que sejam usados como matéria prima em habitações populares. De acordo com a pesquisa, é notável uma preocupação é comum na engenharia civil e apontam um bom caminho a ser seguido, isto é, os resultados parecem válidos e positivos.

Os autores basearam a pesquisa na adição de materiais residuais a fim de diminuir o impacto causado por materiais convencionais. Uma ideia promissora é reutilizar os resíduos sólidos urbanos. Outra, é utilizar os resíduos gerados pela própria construção civil, uma vez que as placas apresentaram resultados satisfatórios em sua estabilização, apesar de precisarem de algumas melhorias. O potencial poluidor de resíduos industriais pode ser transformado em ecológico, pois também apresentaram características que indicam uma possível reutilização em placas cimenticías, principalmente no caso da indústria de papel reciclado. Nelas, os resíduos são gerados em grandes quantidades no Brasil, e em determinados traços nas placas, podem ser uma solução para certos problemas ambientais. A serragem, material que é descartado em grande quantidade, unida aos resíduos de papel e outros elementos cimentícios, também tem potencial de solução sustentável na construção dos elementos de fechamento destinados a moradias de baixo custo.

Os trabalhos encontrados mostram diversas formas de construir essas placas, usando diferentes tipos de resíduos, de maneira satisfatória. Isto é, os testes, como de absorção de água, resistência à tração e compressão, entre outros, apresentaram bons resultados e confirmam a viabilidade técnica desta tecnologia.

Essas pesquisas têm grande relevância, considerando os inúmeros desabrigados e moradias com menos que o mínimo necessário para serem consideradas dignas. $\mathrm{O}$ déficit habitacional do Brasil pode, com esses materiais de baixo custo, ser reduzido. Por este motivo as pesquisas teóricas devem ser continuadas e buscando sempre se manter atualizadas. É importante também intensificar a realização de testes práticos para futuras aplicações dos projetos, como o que pretende ser desenvolvido nos próximos passos desta pesquisa, caminhando assim para a solução, ou ao menos a diminuição de todos os problemas habitacionais e ambientais citados.

\section{R E F E R E N C I A S}

ASOCIACIÓN INTERAMERICANA DE INGENIERÍA SANITARIA Y AMBIENTAL (AIDIS). Directrices para la Gestion Integrada y Sostenible de Residuos Solidos Urbanos en America Latina y el Caribe. São Paulo, 2006. 118 p.

BENATTI, C. T.; LUCAS, Denis. Utilização de resíduos industriais para produção de artefatos cimentícios e argilosos empregados na construção civil. Agronegócios e Meio Ambiente, v. 1, n.3, p. 405-418, set./dez. 2008.

BRAGA JR., BENEDITO P. F. et al. Introdução à engenharia ambiental, o desafio do desenvolvimento sustentável. 2 edição. Abr. 2005.

DEMAJOROVIC, Jacques. Da política tradicional de tratamento do lixo à política de gestão de resíduos sólidos. Revista de Administração de Empresas. São Paulo, v. 35, n.3, p. 88-93 Mai./Jun. 1995.

ARRUDA FILHO N.T.; DANTAS C.P; LEAL A.F; BARBOSA N.P; SILVA C.P; MAVINIEUX, A.V. Resistência mecânica de compósitos cimentícios leves utilizando resíduos industriais e fibras de sisal. v.16, n.8, p.894-902, 2012.

FUWAPE J.A; FABIYI J.S; OSUNTUYI E.O. Technical assessment of three-layered cement-bonded boards produced from wastepaper and sawdust. Volume 27, Issue 11, Pages 1611-1616, 2007.

LACERDA, DANIEL PACHECO; DRESCH, ALINE; ANTUNES, JOSÉ ANTONIO VALLE. Design Science Research - Método de Pesquisa Para Avanço da Ciência e Tecnologia. 2015.

MOURA, J.M.B.M.; MATOS L.F.S. Placas cimentícias à base de resíduos sólidos domiciliares. v. 17, n. 1, 2015.

M. PELEGRINI; I. GOHR PINHEIRO; J.A.B. VALLE. Plates made with solid waste from the recycled paper industry. Volume 30, Issue 2, February 2010, Pages 268-273.

M. CONTRERAS; S.R. TEIXEIRA; M.C. LUCAS; L.C.N. 
LIMA; D.S.L. CARDOSO; G.A.C. DA SILVA;

G.C. GREGÓRIO; A.E. DE SOUZA A. DOS SANTOS. Recycling of construction and demolition waste for producing new construction material (Brazil case-study). Construction and Building Materials.Volume 123, 1 October 2016, Pages 594-600.

SAMPAIO, RF et al. Estudos de revisão sistemática: um guia para síntese criteriosa da evidência científica. vol.1,1 no.1, São Carlos, Jan./Feb, 2007.

SJÖSTRÖM, M.; ÖSTBLOM, G. Decoupling waste generation from economic growth - A CGE analysis of the Swedish case. Ecological Economics, v. 69, Issue 7. 15 May 2010, p.1545-1552.

SOARES, Liliane Gadelha da Costa; SALGUEIRO, Alexandra Amorim; GAZINEU, Maria Helena Paranhos. Educação ambiental aplicada aos resíduos sólidos na cidade de Olinda, Pernambuco - um estudo de caso. Revista Ciências \& Tecnologia. Tecnologia Ano 1, n. 1, julhodezembro 2007. 